\title{
Map Navigation Using a Wearable Mid-air Display
}

\author{
Alexandru Dancu ${ }^{1}$, Mickaël Fourgeaud ${ }^{1}$, Mohammad Obaid ${ }^{1}$, Morten Fjeld ${ }^{1}$, Niklas Elmqvist ${ }^{2}$ \\ ${ }^{1} \mathrm{t} 2 \mathrm{i}$ Lab, Chalmers University of Technology \\ Göteborg, Sweden \\ \{dancu, micfou, mobaid, fjeld\}@chalmers.se
${ }^{2}$ iSchool, University of Maryland
College Park, MD, USA
elm@umd.edu

\begin{abstract}
Advances in display technologies could soon make wearable mid-air displays - devices that present dynamic images floating in mid-air relative to a mobile user-available. Such devices may enable new input and output modalities compared to current mobile devices, and seamlessly offer information on the go. This paper presents a functional prototype for the purpose of understanding these modalities in more detail, including suitable applications and device placement. We first collected results from an online survey identified map navigation as one of the most desirable applications and suggested placement preferences. Based on these rankings, we built a wearable mid-air display mockup consisting of mobile phone, pico projector, and a holder frame, mountable in two alternative ways: wrist and chest. We then designed an experiment, asking participants to navigate different urban routes using map navigation displayed in mid-air. For map navigation, participants ranked wrist-mount safer than chest-mount. The experiment results validate the use of a wearable mid-air display for map navigation. Based on our online survey and experiment, we offer insights and recommendations for the design of wearable mid-air displays.
\end{abstract}

\section{Author Keywords}

Mid-air displays; wearable displays; navigation; prototyping.

\section{ACM Classification Keywords}

H.5.m. Information Interfaces and Presentation (e.g. HCI): Miscellaneous

\section{INTRODUCTION}

Mid-air displays present "floating images in free space" [8, 16]. Here, we define wearable mid-air displays as devices that generate two-dimensional visual content that (i) floats in air, (ii) is positioned relative to a mobile user, and (iii) allows the user to determine the distance of the visual content and to interact with it. Several technologies to create images in mid-air exist, but none have so far reached the general public. However, recent research in holographic displays-initially proposed by Gabor [4] in 1971-has the potential to reduce the cost of manufacturing and may make mid-air displays

Permission to make digital or hard copies of all or part of this work for personal or classroom use is granted without fee provided that copies are not made or distributed for profit or commercial advantage and that copies bear this notice and the full citation on the first page. Copyrights for components of this work owned by others than ACM must be honored. Abstracting with credit is permitted. To copy otherwise, or republish, to post on servers or to redistribute to lists, requires prior specific permission and/or a fee. Request permissions from Permissions@acm.org. MobileHCI '15, August 25 - 28, 2015, Copenhagen, Denmark 2015 ACM. ISBN 978-1-4503-36529/15/08\$15.00 DOI: http://dx.doi.org/10.1145/2785830.2785876

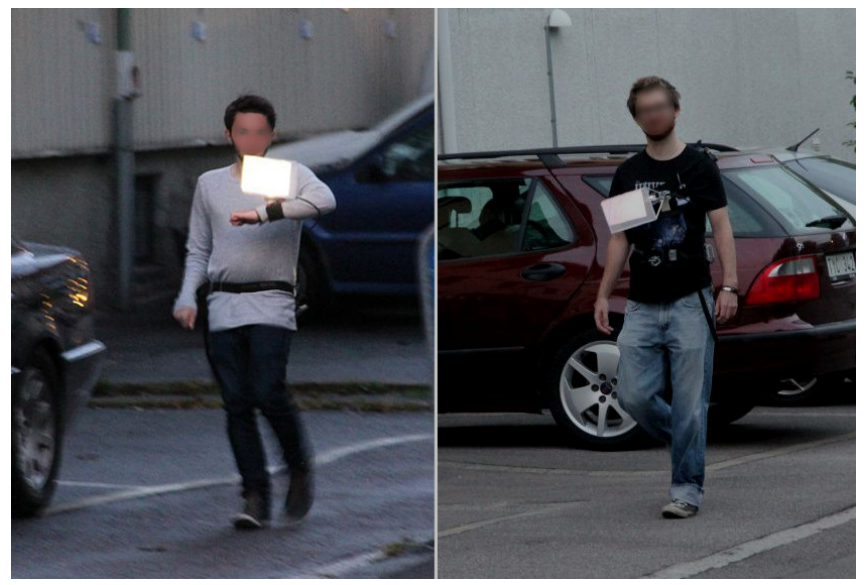

Figure 1. Participants using a prototype of a wearable mid-air display mounted on the wrist (left) and on the chest (right); used with consent.

widely accessible [20]. Mobile wearable holographic devices would yield new opportunities for mobile human-computer interaction not yet fully explored.

We approached the study of wearable mid-air displays by using prototypes-mockups that simulate future technology prior to having access to it-to better understand usage and future interaction with such displays. We also focused on identifying appropriate applications, usage modes, and wearability aspects by studying user interaction with the prototype. We began by conducting an online survey designed to identify suitable tasks for a wearable mid-air display. Based on the rankings, we selected map navigation as the most favorable application to explore. We then designed, built, and evaluated the prototype of a wearable mid-air display for this application. The results validate the utility of our prototype for map navigation and show that wrist-mount was ranked safer than chest-mount by a factor of three.

\section{RELATED WORK}

The background for our work comes from the areas of mid-air display technologies, wearable and mobile projection, wearable on-body projection, and wearability issues. Next, we review these areas in detail.

\section{Mid-air Display Technologies}

Volumetric display technologies, such as swept-volume, or static-volume, "provide all the depth cues humans require" [1]. Pimenta and Santos identify static and dynamic psychologic cues, as well as physiological cues [15]. Static volumetric displays have a volume in which individual voxels (not pixels) can be excited using an energy beam. The 
medium ranges from air to caesium-based vapor, crystal glass, or Plexiglas [5]. Swept-volume include rotating LED arrays, light projected into rotating screens, laser beams with a spinning helix surface, and a varifocal mirror system with a high-speed monitor [5]. Other technologies for mid-air displays include back-projected water particles $[16,17]$ and ultrasound standing waves that can suspend and move particles in the air [13]. Gabor's technique of holograpy allows light wavefronts scattered from a surface to be recorded and then displayed $[4,5]$. However, this approach suffers from the bandwidth limitations of current holographic substrates, high cost, and poor scalability. Smalley et al. [20] propose a method for holographic video displays with a bandwidth 10 times higher than the current state of the art, and which can be manufactured at a cost of $\$ 500[20,5]$. Recent research applications include producing mid-air displays that employ quadcopters [18], water bubbles [19], and fog [10]. Our approach differs in that we propose wearing the display, and displaying information relative to the body and mount.

Wearable and Mobile Displays Projected on Environment Wear-Ur-World (WUW) is a wearable gestural information interface using a head-worn projector and arbitrary surfaces [11]. Interaction techniques have also been prototyped with simulated wrist-worn projectors and wall surfaces [2]. Ota et al. [14] explored 16 body locations for wearing multiple projectors for navigation and a photo-slide show while walking and standing, displaying information on floors. The Ambient Mobile Pervasive Display is a shoulder-mounted projector able to display on surfaces around the environment, the floor, and the hand [23]. Cauchard et al. [3] identify challenges of handheld pico-projectors used on walls, desks, and floors, suggesting that this setting is unsuitable for many tasks. MotionBeam is a mobile projector that couples the movement of the projection to the imagery [22]. ProjectorKit provides technical support for rapid prototyping of mobile projector interaction techniques [21]. Molyneaux et al. [12] developed a handheld projector aware of geometry, displaying content accordingly and enabling multi-touch interaction on arbitrary surfaces. Research on mobile projected displays is dependent on surfaces from the environment. While such schemes have informed our work, they rely on physical surfaces surrounding the user, as opposed to our wearable screen.

\section{Wearable Displays Projected on Body}

Interaction with on-body projected interfaces has emerged after PALMbit [24] and Skinput [7] led to this new research direction. Crowd-funded projects, such as the Ritot projection watch ${ }^{1}$ gained some support, as well as the Cicret Bracelet ${ }^{2}$. Harrison et al. [6] implemented and evaluated a shouldermounted depth sensor-projector system that enables multitouch interaction on the body and on arbitrary surfaces. In contrast, our work projects images in mid-air relative to the user's body. The advantage is that the image can be displayed anywhere in the field of view, but still be controlled directly or indirectly by our body.

\footnotetext{
${ }^{1}$ Ritot, the first projection watch https : //www. indiegogo.com/ projects/ritot-the-first-projection-watch

${ }^{2}$ Cicret Bracelet http: //www. cicret.com
}

\section{DESIGN SPACE AND APPROACH}

The goal of our work was to determine if and how wearable mid-air displays could be used, given wide availability. To focus our exploration, we settled on the following scope:

- Mobile tasks: The main utility of a wearable display is for mobile tasks performed while on the go;

- Outdoor settings: Mobile tasks are often found in outdoor settings; and

- 2D images: We assume 2D (not 3D volumetric) images; mid-air displays are defined as floating images $[8,16]$.

Several technologies exist that fit this scope. We did not consider the recent generation of head-mounted displays such as Google Glass or Microsoft HoloLens since they may be perceived as obtrusive and do not support direct interaction. Similarly, we disregarded the new crop of smart watches because of their small displays which require users to split their attention between the display and the surrounding world. Our approach was informed by an online survey we carried out to gain information on user preferences. Based on our survey results, we created a wearable mid-air display prototype with two alternative body mounts. Based on a pilot study, we improved the prototype and used it within an experiment.

\section{ONLINE SURVEY}

We were inspired by an existing online survey carried out in 2013 in the USA with 4,656 adults, which inquired about their preferred location of a wearable sensor device [9]. From a total of $100 \%$, respondents ranked wrist at $28 \%$, clipped onto clothing at $29 \%$, glasses at $12 \%$, upper arm at $10 \%$, around chest at $6 \%$, embedded in jewelry at $12 \%$, and embedded into clothing at $15 \%$ [9]. However, this existing survey did not study the location of a wearable display. This approach is similar to how we designed our prototype and experiment based on results from an online survey.

We created a survey using the LimeSurvey platform, asking a total of 27 questions organized into six groupings, and completed by university students from online groups. The survey was answered by $37(n=37,100 \%)$ participants $(13$ females) having mean age of 28 (s.d. 8.08). The majority of participants were from the following countries: Canada, Germany, the Netherlands, and Sweden. After an initial demographic query, subjects were offered a brief definition of wearable mid-air display, describing it as one that "consists of computer generated visual content, floating in air." They were then shown a video depicting a person standing and walking outdoors with an animated two-dimensional semi-transparent white rectangle floating in the air relative to his body (Figure 2). This video only showed the actual display position and extent; it did not reveal any information about where on the body a device would be worn, which application was offered by the display, nor how interaction would take place. The video was followed by five sets of questions. The study requested the participant's average time spent per day using a smartphone, and the majority replied with the choice from 1 to 3 hours (54\%). Twenty-five were right-handed, ten lefthanded, and two reported themselves as ambidextrous. 


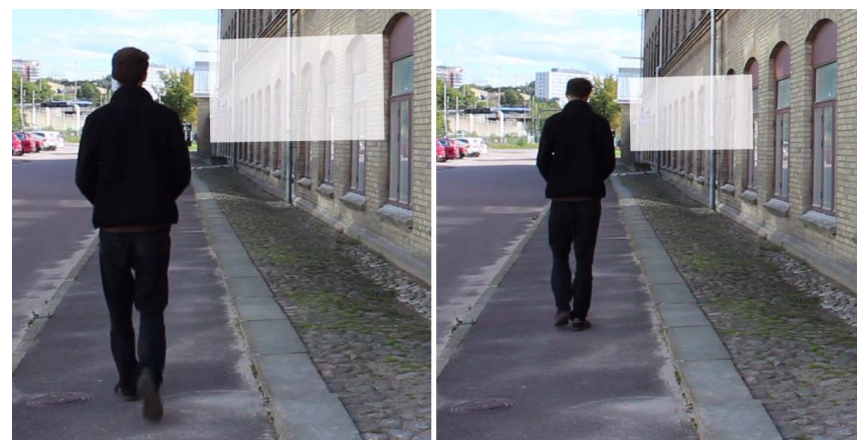

Figure 2. Two successive images from video material illustrating a wearable mid-air display and presented to survey participants.

For most of the questions, multiple responses were accepted. The percentages reported are numbers of choices per alternative by number of participants. Hence, for each question, the sum of percentages may exceed $100 \%$. In those few cases where a single choice was offered, this is stated. Based on data from the survey, shown in Figure 3 (left), the wrist appears to be the most preferred place to wear the device giving a mid-air display, scoring significantly higher than the five alternative parts: head, shoulder, upper arm, chest, and hip. Based on survey data regarding usefulness of wearable midair display applications on a scale from 1 (not useful at all) to 5 (very useful), GPS map navigation had the highest preference score (89.1\% rating 5, Figure 3, right), scoring significantly higher than alternatives such as video chatting $(35.1 \%$ rated $4,27 \%$ rated 5 ), functions of a smartphone ( $40.5 \%$ rated $4,32.4 \%$ rated 5 ), and functions of a laptop (24.3\% rated 2, $37.8 \%$ rated 3 ). Their suggested applications included reading, displaying time, to-do lists, shopping lists, public transportation and local information, camera display, notifications, augmented reality, games for multiple users, and sharing information while having a conversation.

When asked how interested the subject would be in using a wearable device capable of generating a mid-air display providing an application they trusted, the mean answer was (mean 3.84, s.d. 1.09) on a scale from 1 (low interest) to 5 (high interest). For a set of applications we asked subjects "where would you place on your body the wearable device generating the mid-air display?" Starting with video chat, shoulder $(40.5 \%)$ and wrist (37.8\%) were the top candidates. For browsing the web, shoulder $(40.5 \%)$ and wrist $(40.5 \%)$ were the top candidates. For word processor, wrist $(37.8 \%)$ was followed by shoulder (35.1\%) and around chest $(35.1 \%)$. For GPS navigation, head turned out to be the preferred choice $(48.7 \%)$, followed by shoulder $(43.2 \%)$, wrist $(40.5 \%)$, around chest (27\%), and upper arm (24.3\%). On a separate count, we also asked which accessory capable of generating mid-air display subjects would prefer, and the answers were clip-on clothes $(70.3 \%)$, watch $(56.8 \%)$, glasses $(43.2 \%)$, bracelet $(40.5 \%)$, necklace $(29.7 \%)$, earpiece $(18.9 \%)$, ring $(13.5 \%)$, and belt $(13.5 \%)$.

Regarding input and dexterity, we first asked for a preferred input mode for mid-air display, where the alternatives offered gained these multiple score rates: voice control (43.2\%),
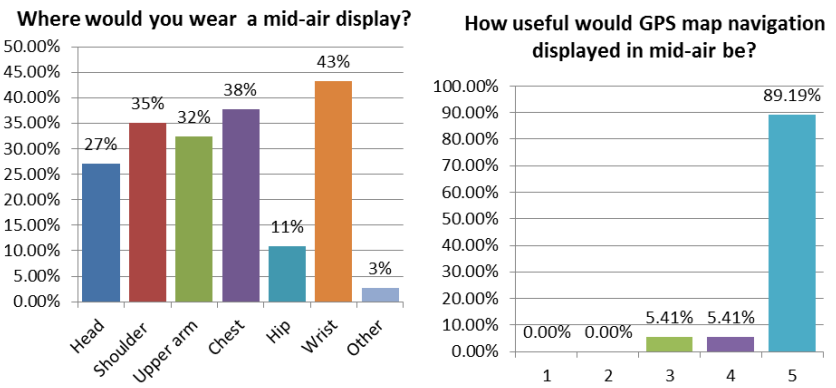

Figure 3. Preferred body positions for mounting a mid-air display device (left); usefulness rating of the map navigation application on a scale from 1 (not useful at all) to 5 (very useful) (right).

eye control (32.4\%), gesture control (pointing or waving in the air) $(67.6 \%)$, touch control (touch on a physical surface) $(59.5 \%)$, and others (brain input was suggested) (13.5\%). When subjects were asked which hand(s) they would use to interact with the mid-air display, they answered left hand $(59.5 \%)$, right hand $(40.5 \%)$, and both hands $(43.2 \%)$. Second, given a display device to be worn on a subject's wrist and asked which wrist they would prefer (single choice only); results were left hand (59.5\%) and right hand (40.5\%). Third, given a display device worn on the subject's wrist, we asked which hand they would use to interact with the display (single choice only); results were left hand $(24.3 \%)$, right hand $(54.1 \%)$, and both hands (21.6\%).

\section{WEARABLE MID-AIR DISPLAY PROTOTYPE}

The prototype consisted of a mobile phone connected to a pico-projector that projects onto a semi-transparent cloth suspended in the air, meant to be worn around the wrist and on the chest. We wanted the projection surface to be both close to the body and within the user's field of view. Following our survey, chest-mount was chosen since it was higher than shoulder and upper arm, but also because this condition would not obstruct limb movement. The projector connects to a mobile phone, powered by an external battery. The modular prototype consists of a holder for the projector connected to a frame that supports a transparent projection surface. Straps and mounts attach it to the wrist or chest. The projector holder, frame, wrist, and chest-mounts were designed in Blender and 3D-printed. The projector holder was designed to securely slide into the wrist- and chest-mount, allowing easy switching of its position. The mobile phone used was an LG Optimus 4X 4D (Android 4.0) with a $1280 \times 720$ resolution. The pico-projector was a PicoMax MX 60 connected to the smartphone via an MHL adapter powered by a battery. The GPS receiver was a Qstarz 818X connected via Bluetooth to the phone, improving the lower location accuracy of the smartphone. The GPS navigation application was GPS Essentials, and routes were designed in the Viking software. The projection surface was a $3 \mathrm{~mm}$ thick semitransparent white fabric with uniform brightness, set inside a $192 \times 196 \mathrm{~mm}$ frame. The screen was attached to the frame using hook and loop straps allowing it to be pulled out, thus preventing any distortions. Two $250 \mathrm{~mm}$ wooden rods, chosen for their rigidity and their low specific weight, were inserted and glued into holes in the frame corners connecting it 

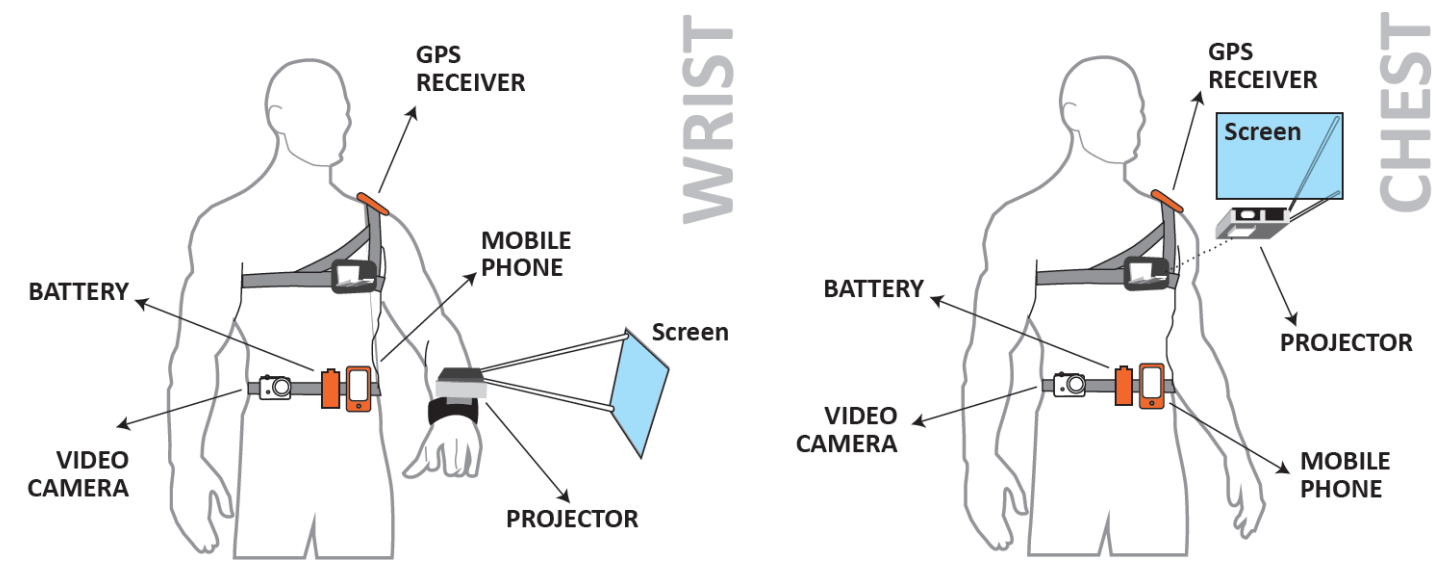

Figure 4. Description and positioning of the two placements of the display on the chest (left) versus on the wrist (right). The actual prototype had the battery and mobile phone attached horizontally at the rear of the belt, shown here in front of the belt for illustration purposes only.

to two holes in the projector holder (Figure 4). The length of these rods determines the distance of the screen from a user's body. The combined weight of holder, projector, rods, and frame was 205 grams. The wrist-mount was attached to a soft cloth hand strap with hook and loop closures, made to be worn on the left wrist. The screen frame and projector holder were oriented to be in the field of view. The chest-mount strap was shaped as a holster, one strap going around the chest and the other over the left shoulder (Figure 4). The weight of the wrist-mount and wrist strap was 35 grams, and the chest strap and Bluetooth GPS weighed 160 grams. The smartphone and battery were attached to the back side of a belt and the video camera in front, with a total weight of 565 grams.

\section{EXPERIMENT}

The task was to navigate two routes displayed on the midair display; one with the wrist, the other with the chest prototype. The experiment was a within-subjects comparative study evaluating the two conditions over two different routes using a balanced distribution of route-device combinations. After each condition, a NASA Task Load Index (TLX) questionnaire was completed. At the end of the second trial, a questionnaire comparing the two mounts was filled out, followed by an interview intended to explain their choices.

\section{Pilot Study}

We conducted a pilot study to confirm whether the prototype was usable for navigation, to get early feedback, improve the prototype, and design the experiment. After initial feedback, we implemented changes to the prototype (Figure 4): (i) an additional light chest strap on which we would attach the GPS receiver (both to improve GPS accuracy and to reduce weight), and (ii) heavier devices (the phone and the battery) moved to the belt. Seven participants (two females); university students aged 18 to 28 years (mean 25 , s.d. 4.16) tested our prototype (five completed the questionnaire). Participants took 30-40 minutes to navigate the two routes, fill out questionnaires, and complete the interview. The time of day was early dusk since direct sunlight affected projector brightness. Paired t-tests for each of the task load questions revealed a significant effect of mount position on frustration, with wrist being lower than chest. The type of device had no significant effect on either other task load aspects or map checking frequency.

\section{Experiment Description}

The main modifications to the experiment after the pilot study were to improve the chest prototype, making it more sturdy, and to improve the comparison questionnaire by adding the options to rate both chest and wrist equally. Another section was added where they could suggest applications, and the routes were changed, making sure they had the same number of turns. We added a question on overall preference, a table for rating the highest task loads from NASA TLX, and questions regarding the preferred hand for wearing and interacting with such a device. For the experiment, we recruited 8 righthanded participants ( 2 females); university students and interaction design professionals aged between 28 and 35 (mean 32.1, s.d. 2.74), no overlap with pilot study above. Mean duration of a route for the wrist condition was 391 seconds (s.d. 45) and for chest 372 seconds (s.d. 26). This data was gathered by recording their travelled route with a video camera mounted on the belt, pointing towards their head (Figure 4).

\section{Experiment Results}

Our results are presented in four categories; preference and usability of wearable device mount position, perceived task workload, interaction, and participant comments.

\section{Preference and Usability of Mount Position}

Figure 5 shows the outcome of the questionnaire. Comparing the two mounts for route attention, $62.5 \%$ chose wrist, $37.5 \%$ chose chest. For road and traffic visibility, $62.5 \%$ chose wrist, $12.5 \%$ chest, and $25 \%$ had no preference. For aid in navigation, $25 \%$ favored wrist, $50 \%$ chest, and $25 \%$ considered them equally helpful. In regard to safety, $75 \%$ chose wrist, $12.5 \%$ chest, and $12.5 \%$ considered them equally safe to use. For ease of use $25 \%$ preferred wrist, $62.5 \%$ chest, and $12.5 \%$ thought them equal. Overall preference was $50 \%$ wrist and $50 \%$ chest. 


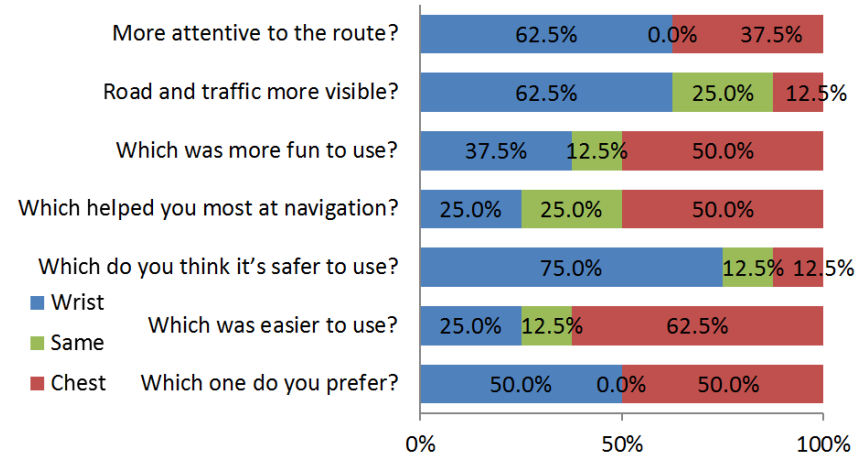

Figure 5. Users system rankings across seven criteria for wrist (blue), no preference (green), and chest (red): attention, road visibility and traffic visibility, navigational aid, safety, ease of use, and overall preference.

\section{Perceived Task Workload}

Paired t-tests for each of the task load questions revealed that the mount location had no significant effect on task load aspects. However, the perceived frequency of checking the map rated on 7-point Likert scale showed a significant effect of mount $(t=-2.3, d f=7, p<.02)$, with wrist being lower (mean 3.7, s.d. 1.4) than chest (mean 5.1, s.d. 1.8) Rating the higher task load revealed the following: (i) for mental demand $37.5 \%$ chose wrist, $37.5 \%$ chest, and $25 \%$ rated them equally; (ii) for physical demand, $50 \%$ chose wrist, $37.5 \%$ chest, and $12.5 \%$ considered them identical; (iii) for temporal demand, $25 \%$ saw no difference between the mounts, $62.5 \%$ chose wrist and $12.5 \%$ chose chest; and (iv) for frustration, $50 \%$ chose wrist, $25 \%$ chose chest, and $25 \%$ rated them equally.

\section{Interaction}

When asked for preferred kind of input with a mid-air display, the most rated option was $50 \%$ for gestures (in the air), followed by $37.5 \%$ for touch (on a physical surface), eye control at $25 \%$, and $12.5 \%$ for voice control. When asked for which hand they would use to interact with the mid-air display, $50 \%$ chose the right hand, and $50 \%$ opted for both hands.

\section{Participant Comments}

During the interview we asked participants to suggest applications they considered useful and to write down other comments. A general suggestion mentioned it would be useful to show "content layered on top of real life," and it should only appear when you want to see it. Others suggested applications from smartphones, such as reminders, e-mail reading, and video chat. Specific applications were suggested, such as motorbiking, comparison shopping, work in the field displaying service diagrams, shared whiteboard for collaboration, text input, and entertainment. One participant noted that navigation would be the most useful, followed by reading e-mail and voice input text messaging while walking, in addition to applications that could offer real-time translation of spoken conversation. Another remarked "It was less disturbing to walk around with the chest-mount, maybe because I got used to having some device sticking out of my body."

\section{DISCUSSION}

The availability of new display technology has the potential of changing human behavior. We explore usage of mid-air displays in a wearable context to support displaying information on the go, safe, and hands-free. The online survey showed a high interest in using a wearable mid-air display (a mean of 3.8 out of 5). Our experiment focused on map navigation and revealed that a wrist-worn mid-air display was ranked safer than chest-worn. This result could hold if the device were mounted on the shoulder, upper arm, or head since some of these locations are in the field of view and are more static as compared to the wrist. When worn on the wrist, users bring the display into their field of view only when information is needed. Unlike palm devices such as smartphones, our wrist-worn prototype only requires changing the hand position without engaging the hand itself (Figure 1). Smart watches are also wrist-worn, but the position of their small screen additionally requires looking down at the wrist. Finally, smart glasses are handsfree, may create the illusion of information floating in air, but are obtrusive and do not allow direct interaction, nor determining the distance to information.

Assuming that safety is an important factor for wearable midair display mounting it on the wrist is recommended. This design choice has implications on interaction with wearable mid-air displays. Alternative orientations of wrist-worn projectors have been studied [2], but their findings cannot be applied directly to wearable mid-air displays, since with projectors users try to achieve visibility by moving the limbs and body according to surfaces in the environment. However, changing the orientation of the light ray parallel or perpendicular to the forearm has potential benefits. That is, parallel orientation can leave room for finger input of the same hand, whereas a perpendicular mount requires the other hand for input. The online survey listed preferred input methods as pointing, touch, voice, and eye control, in that order. Future work could include studying pointing and touch input for horizontal and parallel mounts, as well as different transparency levels and screen sizes.

Beyond map navigation, many additional applications were suggested, out of which the real-time translation system was particularly interesting. The information displayed in mid-air opens up a range of applications that have not yet been possible. Our prototype can help explore interaction with mid-air displays, suggest where to mount it when taking into account road and traffic visibility, navigate a map, and suggest the preferred applications for outdoor usage. The wearable mid-air display prototype is easy to implement and safe to use, and could help researchers set up experiments and evaluate human factors regarding such settings, interactions, and future ways of using information.

\section{CONCLUSION}

We have presented a qualitative exploration of the design space of wearable mid-air displays for mobile settings. Our findings from an online survey showed a significant preference for map navigation, which led us to focus on this application. The survey showed a preference for mounting on the wrist or the chest, which led us to build a functional prototype enabling us to compare these alternative mounting positions. We ran an experiment comparing these two conditions in an 
outdoor setting, giving us novel insights that can inform the design of future systems. The wrist-mounted solution was ranked safer than chest-mounted solution by a factor of three. Our results validated the use of such a mid-air display for map navigation on the go. While development of mid-air technology may soon be available, our prototype shows how such hands free devices may allow for new kinds of interaction, and further exploration into new uses of information in midair.

\section{Acknowledgments}

We thank Waldir Pimenta, Péter Tamás Kovács, Barrie James Sutcliffe, Adviye Ayça Ünlüer, and Philippa Beckman. This work was supported by the EU FP7 People Programme (Marie Curie Actions) under REA Grant Agreement 290227.

\section{REFERENCES}

1. Balakrishnan, R., Fitzmaurice, G. W., and Kurtenbach, G. User interfaces for volumetric displays. Computer 34, 3 (2001), 37-45.

2. Blasko, G., Coriand, F., and Feiner, S. Exploring interaction with a simulated wrist-worn projection display. In Proceedings of the IEEE Symposium on Wearable Computers (2005), 2-9.

3. Cauchard, J. R., Fraser, M., Han, T., and Subramanian, S. Steerable projection: exploring alignment in interactive mobile displays. Personal and Ubiquitous Computing 16, 1 (2012), 27-37.

4. Gabor, D., Kock, W. E., and Stroke, G. W. Holography. Science 173, 3991 (1971), 11-23.

5. Geng, J. Three-dimensional display technologies. Advances in Optics and Photonics 5, 4 (2013), 456-535.

6. Harrison, C., Benko, H., and Wilson, A. D. OmniTouch: wearable multitouch interaction everywhere. In Proceedings of the ACM Symposium on User Interface Software and Technology (2011), 441-450.

7. Harrison, C., Tan, D., and Morris, D. Skinput: appropriating the body as an input surface. In Proceedings of the ACM Conference on Human Factors in Computing Systems (2010), 453-462.

8. Hoshi, T., Takahashi, M., Nakatsuma, K., and Shinoda, H. Touchable holography. In ACM SIGGRAPH 2009 Emerging Technologies (2009), 23.

9. J. P. Gownder et al. Building A Fitter Business With Wearable Technology. Forrester Research, Inc., 2014.

10. Martinez Plasencia, D., Joyce, E., and Subramanian, S. MisTable: reach-through personal screens for tabletops. In Proceedings of the ACM Conference on Human Factors in Computing Systems (2014), 3493-3502.

11. Mistry, P., Maes, P., and Chang, L. WUW-wear Ur world: a wearable gestural interface. In Extended Abstracts of the ACM Conference on Human Factors in Computing Systems (2009), 4111-4116.

12. Molyneaux, D., Izadi, S., Kim, D., Hilliges, O., Hodges, S., Cao, X., Butler, A., and Gellersen, H. Interactive environment-aware handheld projectors for pervasive computing spaces. In Proceedings of the International Conference on Pervasive Computing (2012), 197-215.
13. Ochiai, Y., Hoshi, T., and Rekimoto, J. Pixie dust: Graphics generated by levitated and animated objects in computational acoustic-potential field. ACM Transactions on Graphics 33, 4 (July 2014), 85:1-85:13.

14. Ota, S., Takegawa, Y., Terada, T., and Tsukamoto, M. A method for wearable projector selection that considers the viewability of projected images. Computers in Entertainment (CIE) 8, 3 (2010), 17.

15. Pimenta, W., and Santos, L. P. A comprehensive taxonomy for three-dimensional displays. In Proceedings of the International Conference on Computer Graphics, Visualization and Computer Vision (2012), 139-146.

16. Rakkolainen, I. How feasible are Star Wars mid-air displays. In Proceedings of the International Conference on Information Visualization (2007), 935-942.

17. Rakkolainen, I., and Palovuori, K. Walk-thru screen. In Proceedings of Electronic Imaging, International Society for Optics and Photonics (2002), 17-22.

18. Schneegass, S., Alt, F., Scheible, J., Schmidt, A., and $\mathrm{Su}, \mathrm{H}$. Midair displays: Exploring the concept of free-floating public displays. In Extended Abstracts on the ACM Conference on Human Factors in Computing Systems (2014), 2035-2040.

19. Seah, S. A., Martinez Plasencia, D., Bennett, P. D., Karnik, A., Otrocol, V. S., Knibbe, J., Cockburn, A., and Subramanian, S. Sensabubble: a chrono-sensory mid-air display of sight and smell. In Proceedings of the ACM Conference on Human Factors in Computing Systems (2014), 2863-2872.

20. Smalley, D., Smithwick, Q., Bove, V., Barabas, J., and Jolly, S. Anisotropic leaky-mode modulator for holographic video displays. Nature 498, 7454 (2013), 313-317.

21. Weigel, M., Boring, S., Steimle, J., Marquardt, N., Greenberg, S., and Tang, A. ProjectorKit: easing rapid prototyping of interactive applications for mobile projectors. In Proceedings of the ACM Conference on Human-Computer Interaction with Mobile Devices and Services (2013), 247-250.

22. Willis, K. D., Poupyrev, I., and Shiratori, T. MotionBeam: a metaphor for character interaction with handheld projectors. In Proceedings of the ACM Conference on Human Factors in Computing Systems (2011), 1031-1040.

23. Winkler, C., Seifert, J., Dobbelstein, D., and Rukzio, E. Pervasive information through constant personal projection: The ambient mobile pervasive display (AMP-D). In Proceedings of the ACM Conference on Human Factors in Computing Systems (2014), 4117-4126.

24. Yamamoto, G., and Sato, K. Palmbit: A palm interface with projector-camera system. In Adjunct Proceedings of Ubicomp, vol. 2007 (2007), 276-279. 